\title{
Las TIC en la UPEL visto desde sus productos académicos
}

\author{
ICT in UPEL seen from its Academic Products
}

\begin{tabular}{l} 
Belkys Guzmán \\
belkys.juliana.guzman@gmail.com \\
Código ORCID: 0000-0002-8141-5990 \\
Universidad Pedagógica Experimental Libertador, Caracas, Venezuela \\
$\begin{array}{l}\text { Santiago Castro } \\
\text { castrosantiago2015@gmail.com } \\
\text { Código ORCID: 0000-0003-2848-0870 } \\
\text { Universidad Pedagógica Experimental Libertador, Caracas, Venezuela }\end{array}$ \\
\hline
\end{tabular}

Artículo recibido en febrero 2019 / / Arbitrado en marzo 2019 / Publicado en mayo 2019

\section{Resumen}

El artículo pretende construir un hito basado en hechos claves y fundamentales dentro del ámbito o contexto de la incursión en la UPEL de las Tecnologías de Información y Comunicación (TIC). La investigación pormenorizada fue de tipo documental. Conformó el estado del arte de las TIC en la UPEL, se basó en la descripción, análisis e interpretación de sus publicaciones. Este compendio incluyó las prioridades y definiciones de las políticas de incorporación de las TIC en la formación docente, así como la interpretación de los documentos que la universidad hace explícitos en relación con este conocimiento. El impacto de las TIC en la comunidad upelista, los conceptos, tipos, características que maneja o han manejado, los usos que se les da y que proponen en la UPEL para la formación y apropiación de las Tecnologías de Información y Comunicación, y cuáles fueron sus inicios en la universidad fueron aspectos relevantes de los hallazgos y conclusiones del estudio.

\footnotetext{
Abstract

The article aims to build a milestone based on key and fundamental facts within the scope or context of the incursion into UPEL of Information and Communication Technologies (ICT). The detailed investigation was documentary. He conformed the state of the art of ICT at UPEL, based on the description, analysis and interpretation of his publications. This compendium included the priorities and definitions of the ICT incorporation policies in teacher training, as well as the interpretation of the documents that the university makes explicit in relation to this knowledge. The impact of ICT in the upelista community, the concepts, types, characteristics that it manages or have managed, the uses that are given and that are proposed at UPEL for the training and appropriation of Information and Communication Technologies, and which were his beginnings at university were relevant aspects of the study's findings and conclusions.
}

\section{Palabras clave:}

Tecnología de Información y Comunicación; educación; productos académicos
Keywords:

Information and Communication Technology; education; academic products 
INTRODUCCIÓN

Las culturas y costumbres del ser humano, en cualquier parte del mundo, se integran al difuminarse las fronteras geográficas a través de los diferentes dispositivos y herramientas tecnológicas. Las economías mundiales se acoplan por medio del comercio, el intercambio de información; la transferencia de tecnología a través de sus frontera, desdibujadas o no, es inmensa. Los flujos financieros y la movilidad de la mano de obra calificada se extrapolan a tal punto que se forman en un país como el nuestro y son deseados en el exterior por su preparación y dedicación, es por ello que ningún individuo, grupo, país o nación puede estar ajeno a sus efectos, características, tendencias, potencialidades, mitos $\mathrm{y}$ realidades en todos los ámbitos de la vida, entre ellos el trabajo y la educación (Guzmán, 2005, 2008).

La irrupción de los productos de la simbiosis Ciencia-Tecnología, en todos los ámbitos del conocimiento y contextos del quehacer humano, señalan la vía para tomar decisiones, resolver problemas, para mejorar la vida, y en algunos casos el ambiente, con los efectos positivos o no, propias de cada sujeto, nación o país, lo que implica una variación en la calidad de vida en el mundo (Guzmán, 2013).

Es por ello que las distintas generaciones deben prepararse con nuevas competencias básicas y genéricas además de conocimientos, valores e ideales que le permitan optar de la mejor manera por un trabajo productivo y ser un activo para su empresa, estos talentos humanos deben desarrollar aptitudes y actitudes positivas y estar adaptados.

En este contexto mundial y haciendo un recorrido por la historia de la universidad en los últimos años, se ha observado cómo la tecnología ha incursionado lentamente en las aulas y se ha quedado en ellas a pesar de la natural resistencia al cambio por desconocimiento, falta de capacitación y actualización de los docentes y la comunidad educativa en general.

Inicialmente con el uso de medios tradicionales como son rotafolios, diapositivas, fotografías, radio y televisión. Al respecto refiere Guzmán (2016) que en 1936 inician las labores del Instituto Pedagógico Nacional (IPN), el instituto de más larga data de los que conforman la UPEL. En el inicio de su funcionamiento se fijan las normas para el establecimiento de los cursos de formación de los profesores de educación secundaria y normal que en el artículo número 5 se colocan las bases que sirven de antecedentes a la organización y administración de los procesos de enseñanza y aprendizaje como son los medios audiovisuales en el IPN. Las normas según Albornoz (1986) expresan que:

Los alumnos deberán adquirir, además, mediante cursos auxiliares $\mathrm{u}$ otros medios las siguientes habilidades: a) Dibujo de Ilustración didáctica; b) Manejo de aparatos de proyección fija y animada, empleo didáctico de los mismos y c) uso, preparación y reparación del material de enseñanza en sus respectivas especialidades. (p.19)

El mismo autor refiere que se pretendía "contribuir a la tecnificación de la enseñanza mediante la realización de programas mínimos de ayuda para la escuela primaria, la enseñanza media y la Educación normal" (Guzmán, 2016, p. 377), llegándose el caso de que hay autores que consideran que el pizarrón (verde, negro o ahora acrílico), el puntero con algunas modificaciones permanecen en las aulas como los recursos más utilizados por el docente hasta el punto de considerarlos imprescindibles e 
inseparables del educador y del educando (Guzmán, 2013).

Volviendo a la actualidad, llama la atención que en la universidad y otros lugares personas que leen algo en sus celulares, otros en tabletas y/o laptop examinan libros, separatas $\mathrm{u}$ hojean periódicos, se actualizan con algún video o audio. Revisan noticias, informaciones varias y en algunos casos en menor cuantía revisan sus aulas virtuales, corrigen y reenvían trabajos a sus grupos académicos.

Un sinnúmero de personas de diferentes edades, culturas y grados académicos andan por el mundo sin papel y llevan un lápiz o un bolígrafo que no utilizan, una tiza o un marcador que se usará más tarde en la clase o para escribir recordatorios. $\mathrm{Y}$ en tiempos mejores del país siempre se lleva el celular cada vez más sofisticado, porque no solo puede llamar sino plasmar con él momentos que solo lo podía hacer con una cámara, detener el tiempo e incluso convertirse en cronista de situaciones que le afectan.

Además, las diferentes aplicaciones les permiten a los individuos ubicarse en la dirección o ruta a seguir, reír, jugar y los mantiene unidos a sus allegados o seres queridos a través de llamadas, video llamadas, mensajes de voz o de texto, que puede escribir o leer por SMS o por WhatsApp.

A través de diferentes tipos de dispositivos tales como computadoras, teléfonos celulares, tablets y laptops, que permitan tener acceso a Internet, los niños, jóvenes y adultos pueden desarrollar sus habilidades, destrezas y desenvolverse fácilmente en la red; con tan solo tener una cuenta y participar en redes sociales generales o profesionales, que permiten mantener relaciones en diferentes ámbitos (Ollarves, Hernández y Pirela, 2018).

La apropiación y uso de las TIC en cualquier ámbito, implica la unión, el progreso y la valoración de escenarios didácticos, socioculturales, curriculares y comunitarios susceptibles de ser utilizadas en los diferentes espacios y profesiones. El uso de estas en las organizaciones ya sea empresarial o educativa beneficia $y$ promueve la interacción de sus trabajadores, automatización de los procesos, desarrollo de nuevos negocios, integración de los procesos en red; existen organizaciones que enfrentan obstáculos de diferente tipo; materiales y equipos no actualizados, personas no capacitadas o desactualizadas.

Por ello deben invertir en su capital humano y en plataforma actualizada. Desafortunadamente, cada vez que se invierte en plataforma tecnológica en la universidad para actualizar equipos dañados $\mathrm{u}$ obsoletos, los amigos de lo ajeno se las roban y dejan el eco de la frustración y la misma necesidad que se tenía antes de adquirirlos.

Cuando se habla en la UPEL de las TIC siempre hay uno que otro "experto" que señala que para la UPEL ha sido prioridad su incorporación en las aulas y además que sus docentes están apropiados de manera significativa de las TIC. Es una creencia de amplia magnitud, pues, por ejemplo, en el Plan de desarrollo de talento humano para los docentes no están contemplados cursos de actualización en esta área, pero las TIC son incorporadas en el diseño de sus cursos y de esta manera son promovidas de manera efectiva en sus estudiantes. Además, que estos últimos son nativos digitales por lo que se manejan con propiedad las TIC. Desafortunadamente la realidad concreta muestra otra cosa.

En el marco de esta problemática, surgieron las siguientes preguntas: ¿qué se ha publicado en la universidad al respecto?, ¿responden los productos académicos a estas creencias o muestran lo contrario?, ¿de qué tratan?, y ¿cuáles aspectos de las TIC consideran importantes? 
Lo que inquietó a los investigadores y captó el interés es la información contenida en los productos académicos relacionada con los saberes y conocimientos generados en la Universidad Pedagógica Experimental Libertador (UPEL).

El significado otorgado a las TIC como eje curricular le transfiere la caracterización y peculiaridad de matizar todo el diseño de la UPEL; al mismo tiempo que brinda la oportunidad del desarrollo de un marco conceptual y metodológico para articular las TIC a la formación inicial y permanente del docente venezolano, tal como lo expresa el Vicerrectorado de Docencia (2008) en la propuesta metodológica de trabajo para la construcción colectiva de la estructura del currículo de pregrado.

Estos aportes significativos emergentes sobre las TIC en los testimonios de los docentes, requieren ser considerados en este momento coyuntural de transformación universitaria por el cual atraviesa la UPEL, en virtud de que representan una necesidad sentida en las voces de los actores sociales que forman parte de este proceso (Oviedo, 2010).

Este estudio pretendió construir algunos hechos claves y fundamentales dentro del ámbito o contexto de la incursión en la UPEL de las Tecnologías de Información y Comunicación (TIC). Es un estudio que conforma el estado del arte de las TIC en la UPEL basado en la descripción, análisis e interpretación de sus publicaciones. Este compendio incluye las prioridades y definiciones de las políticas de incorporación de las TIC en la formación docente e interpretar la información explícita en los documentos existentes en la universidad en relación con este conocimiento.

\section{Las TIC en la UPEL}

Las TIC y la globalización son considerados como impulsores de los cambios y de las nuevas necesidades de formación y de autorrealización de los ciudadanos. Exigen respuestas que debe dar la educación en estos tiempos de cambio y transformaciones y son las Tecnologías de Información y Comunicación las que hacen que el ser humano las considere imprescindibles para todo propósito (Casado, Castro y Guzmán, 2007).

Las características de las TIC permiten almacenar, recuperar, procesar, reportar, presentar, difundir la información (audios, videos, sonidos, archivos de todo tipo y en el formato que deseamos) para usarlo en investigación, docencia, extensión, actualización y capacitación con la finalidad de generar impacto en la comunidad upelista.

Los conceptos, tipos. características que maneja o han manejado los autores de los diferentes productos académicos, en relación con las Tecnologías de Información y Comunicación, los usos que se les da y que proponen en la UPEL para la formación inicial y continua, capacitación y apropiación de las mismas, por arte de la comunidad educativa incluyendo sus egresados, son aspectos que acercaron a interrogantes como: ¿cuáles fueron sus inicios?, ¿cuáles fueron las necesidades visualizadas?, y ¿cómo han sido enfrentadas en la Universidad? (Castro y Guzmán, 2001; Dilone, 2019; Castillo, 2019; Tovar, 2019 y Guzmán, 2018).

Ahora bien, el Estado tiene la responsabilidad de ofrecer a la población una educación de calidad y con pertinencia social, cultural y económica, en concordancia con las exigencias de la sociedad de la información y el conocimiento (Ruiz Bolívar, 2014), y ha generado una serie de políticas y decretos, pero con poca idea de la capacitación de los usuarios directos.

$\mathrm{Al}$ respecto Castro (2009) reflexiona acerca de la ausencia de políticas educativas que faciliten la gestión del conocimiento almacenado y producido por la comunidad, que permita adecuarse a los diferentes 
escenarios. Esto ha provocado que la escuela no realice estudios prospectivos que permitan la anticipación y la visualización de generación de los cambios requeridos para adecuarse a las condiciones del contexto nacional y mundial. En consecuencia, no hay respuestas oportunas ni capacidad de anticipación; lo que genera poca vinculación de la Universidad con la sociedad y el Estado. Es por ello que la escuela se desfasa de los adelantos, de lo cual no escapa la UPEL y la incorporación efectiva de las TIC.

\section{Políticas de la UPEL}

La incorporación de las TIC en las instituciones es una política del Estado venezolano y también lo debe ser en las universidades. Los administradores de éstas deberían entender que es una parte indisoluble de cualquier política general. Por ello se sugirió promover en las instancias el aprovechamiento de las TIC.

La UPEL tiene políticas de docencia, investigación y extensión, las cuales se revisaron durante la investigación para ver cómo figura la información relacionada con TIC. También se analizó ese tema en los planes de desarrollo que duran varios años. En relación con las políticas de: Docencia (UPEL, 2000 y UPEL, 1985), de Investigación (UPEL, 1989 y UPEL., 1985) y Extensión (UPEL, 1985 y UPEL, (1997), no hacen ninguna referencia a TIC solo en las de docencia se especifica una política de medios, en el caso de Extensión (UPEL, 2004), en la política 1 y la estrategia 16 habla específicamente de TIC. Esta labor se organizó y se intensificó en las Jornadas de Extensión del mes de junio del año 2003.

Para generar servicios y productos que respondan a las necesidades, requerimientos y expectativas académicas, culturales, informativas, tecnológicas, deportivas y recreativas de la comunidad se requieren de un conjunto de estrategias como:
1. Apoyo a iniciativas de producción tecnológica, científica, académica, por parte de la comunidad.

2. Fomento del uso de las Tecnologías de Información y Comunicación (TIC), como aporte a la sociedad.

3. Realización de encuentros académicos, culturales, informativos, tecnológicos, deportivos $y$ recreativos intra $y$ extrainstitucionales.

4. Realización de acciones orientadas al uso efectivo de los recursos humanos, materiales y tecnológicos para el desarrollo y beneficio de la comunidad.

5. Desarrollo de nuevos canales de comunicación que permitan promocionar y difundir los productos, servicios y publicaciones especiales de Extensión.

En cuanto a los reglamentos de Extensión, se debe acotar que el de 1985 y 1997 no incluía información relacionada con el área específica. Solo el Reglamento de Extensión UPEL del año 2003 expresa en su Artículo 17 que: "El Programa de Tecnologías de Información y Comunicación integra las actividades necesarias para promover e incentivar la incorporación y el uso efectivo de las herramientas tecnológicas (impresas/audiovisuales/informáticas) para el desarrollo de la Extensión Universitaria" (Universidad Pedagógica Experimental Libertador, s.n).

La UPEL ha reconocido la incursión efectiva de las TIC para la optimización de los procesos educativos actuales en diferentes trabajos de grado, tesis doctorales y en los planes de desarrollo 2007-2011, 20072013, 2014-2018 concebidos bajo el enfoque del modelo de Gerencia Estratégica, los cuales se rigen por los siguientes cinco ejes 
estratégicos: Formación, Investigación, Integración con la Sociedad, Talento Humano y Gestión.

Todo el sistema educativo, desde sus políticas, el currículo, los docentes, el alumno y los procesos de enseñanza y de aprendizaje, se ve impactado transversalmente por las TIC ya que éstas potencian cada uno de los elementos del sistema instruccional y educativo, generando nuevas necesidades y respondiendo a los requerimientos de los escenarios educativos mediante la creación de ambientes de aprendizaje que consideren la atención a la diversidad funcional, la interculturalidad, la ruralidad y el uso de las TIC (Tecnologías de Información y Comunicación).

Es por ello que es imperante introducir cambios en el sistema educativo, desde sus políticas hasta los contenidos más pequeños, realizar las intervenciones necesarias en los elementos esenciales del currículo y en general en los elementos del sistema instruccional, a través de sus subsistemas: elementos directrices (fines, propósitos, metas) docentes, estrategias, recursos y medios de instrucción, entre otros.

En los elementos directrices; propósitos, fines, competencias u objetivos, se espera que se incluyan contenidos relacionados con el uso, apropiación e importancia de las TIC en todos los ámbitos, tanto a nivel personal como profesional, en el área de investigación y docencia para que pueda promover su uso en sus futuros educandos, adaptándolos al mundo cambiante de hoy.

Un ejemplo palpable de la incorporación de las TIC en la UPEL-IPC fue la experiencia vivida por De Marco (2002) y sus compañeros de Departamento en el año 2001, donde se realizó un proceso de capacitación de los docentes más nóveles en cuanto al uso de la herramienta PowerPoint e Intranet, la apertura de un correo electrónico personal y uno del Departamento. Se capacitaron a cinco (5) profesores y luego éstos a su vez, tenían que capacitar a cinco (5) de sus compañeros, es decir, un efecto cascada.

Al cabo de un semestre, todos los profesores del Departamento de Tecnología Educativa, estaban entrenados en el uso de dichas competencias. Se hace necesario destacar que el desarrollo del espíritu crítico permitió no despreciar ni sobrevalorar el uso de la tecnología. Posteriormente, profesores de otros departamentos solicitaron la incorporación a este tipo de cursos para desarrollar sus competencias y así sumarse a la innovación y estableciéndose como objetivo la permanente actualización y la elevación de la calidad educativa.

Hay que comprometerse con la enseñanza bien planificada y ejecutada independientemente de su modalidad, siempre centrada en el estudiante, considerar que debe difundirse y estar por encima de lo perceptible, sensible y eficaz para aproximarnos darle paso a la creatividad, a la imaginación a lo no esperado con base en contextos, espacio y tiempo, con la mediación de las TIC.

De lo expuesto anteriormente, se enfatiza el desafío de abordar una sociedad del conocimiento sellada por el uso de las TIC que apunta a una redimensión del conocimiento, la información y el aprendizaje dentro de los procesos de enseñanza y aprendizaje del ciudadano de hoy que requiere el país y el mundo globalizado.

En cuanto a las tecnologías, estas pueden fortalecer los espacios de instrucción educativa, haciéndolo más participativo, multidisciplinario e investigativo para que el educando tenga un rol activo (Guzmán, 2005, 2008).

Las TIC son un factor de desarrollo debido a sus características y bondades. Poseen innumerables cualidades y ventajas que implican la necesidad de formación, 
tanto del que enseña como en el aprendiz, porque trae consigo cambios significativos en los roles de docentes y alumnos, conduciendo a un proceso más centrado en el aprendizaje interactivo. Las TIC potencian la creación de espacios comunicativos que permitan experiencias formativas para el fomento de la participación, el uso de estrategias y medios innovadores que potencien el aprendizaje por parte del estudiante y del docente.

Guzmán (2012) y Niño (2017) coinciden en señalar que la apropiación de las TIC por parte de docentes y estudiantes en el desarrollo de las actividades académicas de las universidades venezolanas, involucra aspectos tales como:

1. El soporte del estado en los aspectos económicos y de soporte tecnológico.

2. El andamio organizacional en el diseño de espacios y actividades de capacitación, actualización y formación permanente y asesoramiento en los aspectos relacionados al uso de las TIC.

3. La actitud positiva e intencionalidad del docente en la capacitación y participación para el uso y apropiación de las TIC en sus actividades académicas.

4. El intercambio entre docentes, para la formación de grupos de fortalecimiento en aspectos académicos y tecnológicos.

Al respecto señalan Villarroel, Carpio y Castillo (2014) que las TIC en la universidad deben superar su utilización como herramienta transmisora de información, pues para convertirse en un elemento transformador dentro del recinto académico, también se debe potenciar la utilización de la información electrónica a todos los niveles: desde la comunicación entre las personas que trabajan en las instituciones, a la gestión y administración universitaria, pasando por las actividades relacionadas con la investigación, y tomando en cuenta su utilización en la formación.

Señala Guzmán (2016) que los contextos educativos mediados por las TIC, en los cuales, la educación, el conocimiento, el saber, la información y la comunicación presentan significados diversos y conducen al desarrollo del pensamiento autónomo, reflexivo y transformador del ser humano y, de igual manera, a la construcción del saber para mejorar la calidad de vida, retan a la búsqueda de visiones más complejas en las que tengan cabida otros espacios de intercambios entre los individuos por vía del redimensionamiento del lenguaje.

El problema es vincular el contexto social y el contexto educativo con el objeto de comprender los aspectos, conceptuales, actitudinales y procedimentales, las experiencias previas y el proceso de formación. Sin embargo, no se contó con la estructura académica en los planes de estudios, ni de gestión, ni de infraestructura para integrar la tecnología, desde una perspectiva pedagógica. (Beltrán, 2017).

Para el año 2012 en el Siso Martínez, extrapolable a toda la UPEL, se determinó que muy pocos docentes y estudiantes poseen computadoras en sus aulas, cubículo ni en el laboratorio de informática, por lo cual no tienen acceso a Internet en el lugar de trabajo.

En su totalidad, los docentes manifestaron tener interés en aprender a utilizar las TIC para realizar actividades como: gestionar datos académicos, tener acceso a información para preparar las clases, presentar información, preparar exámenes, utilizar medios didácticos y comunicarse con los estudiantes y colegas. 
MÉTODO

El presente estudio fue de tipo documental basado en la revisión de fuentes relacionadas con el estado del arte de lo que diversos autores han aportado en relación con atributos educativos alcanzados por las TIC. La finalidad fue revisar los rasgos y posibilidades de la herramienta para reconstruir una caracterización que ofrezca renovados referentes de utilidad docente, definiciones, ejemplos, sus tipos y su utilización en la promoción y difusión.

La metodología empleada se basó en el registro descriptivo en una matriz de análisis por parte de los investigadores y se realizaron varias fichas digitales en una bibliografía anotada que registran la información proveniente de las fuentes de la investigación. Por otra parte, se trabajó la técnica del análisis de contenido, que según Yegres (2013) citado por Rodríguez (2016) consiste en describir la importancia que tienen los textos hablados o escritos, proceso que logró interpretar, relacionar, organizar, sistematizar y presentar los hallazgos de la investigación.

Los datos originales o primarios fueron recogidos en forma directa de la realidad, los mismos son categorizados de acuerdo al Proceso de Teorización propuesto por Martínez (2010) citado por López (2016). Estuvo comprendido por los procesos de categorización, estructuración, contrastación y teorización.

La población estuvo conformada por todos los documentos académicos generados en la UPEL estén publicados o no y la muestra la conformaron las tesis, trabajos de grado, artículos en revistas arbitradas y en algunos casos indexadas relacionadas con TIC y los documentos legales de la Universidad Pedagógica Experimental Libertador.

La Tabla 1 resume parte de los documentos analizados:

Tabla 1. Muestra de documentos

\begin{tabular}{ll}
\hline Número & Documentos \\
\hline 9 & Trabajos de grado de maestrías \\
11 & Tesis doctorales y 3 trabajos de ascenso \\
14 & Políticas de docencia (UPEL, 2000) \\
8 & Políticas de docencia (UPEL, 1985a) \\
10 & Políticas de investigación (UPEL, 1989) \\
10 & Políticas de investigación (UPEL, 1985b) \\
5 & Políticas de extensión (UPEL, 1985c) \\
9 & Políticas de extensión (UPEL 1997) \\
6 & Políticas de extensión (UPEL, 2004) \\
\hline
\end{tabular}

La Tabla 2 muestra los documentos analizados, es de acotar que las revistas Investigación y Educare publicadas por los Institutos más antiguos de la UPEL son los que han recibido, arbitrado y publicado más artículos relacionados con las TIC. 
Tabla 2. Artículos de revistas publicadas por la UPEL

\begin{tabular}{|c|c|c|}
\hline $\begin{array}{c}\mathrm{N}^{\circ} \text { de art } \\
\text { publicados }\end{array}$ & $\begin{array}{c}\text { Nombre de la revista dirección } \\
\text { electrónica }\end{array}$ & $\begin{array}{c}\text { Pública UPEL Subdirección de } \\
\text { Investigación y Postgrado del instituto }\end{array}$ \\
\hline 10 & $\begin{array}{l}\text { Dialéctica } \\
\text { http://revistas.upel.edu.ve/index.php/di } \\
\text { alectica }\end{array}$ & Pedagógico Rural Gervasio Rubio \\
\hline 12 & $\begin{array}{l}\text { Dialógica } \\
\text { http://revistas.upel.edu.ve/index.php/di } \\
\text { alogica }\end{array}$ & $\begin{array}{l}\text { Pedagógico de Maracay "Rafael Alberto } \\
\text { Escobar Lara }\end{array}$ \\
\hline 34 & $\begin{array}{l}\text { Educare } \\
\text { http://revistas.upel.edu.ve/index.php/e } \\
\text { ducare }\end{array}$ & $\begin{array}{l}\text { Pedagógico "Luis Beltrán Prieto Figueroa" } \\
\text { de Barquisimeto. }\end{array}$ \\
\hline $\begin{array}{l}56+3 \\
\text { reseñas }\end{array}$ & $\begin{array}{l}\text { Revista de Investigación } \\
\text { http://revistas.upel.edu.ve/index.php/re } \\
\text { vinvest }\end{array}$ & Pedagógico de Caracas \\
\hline 2 & $\begin{array}{l}\text { Entretemas } \\
\text { http://revistas.upel.edu.ve/index.php/e } \\
\text { ntretemas }\end{array}$ & Pedagógico Rural El Mácaro. \\
\hline 4 & $\begin{array}{l}\text { Sapiens } \\
\text { http://revistas.upel.edu.ve/index.php/sa } \\
\text { piens }\end{array}$ & $\begin{array}{l}\text { Pedagógico de Miranda José Manuel Siso } \\
\text { Martínez }\end{array}$ \\
\hline 21 & $\begin{array}{l}\text { Sinopsis Educativa } \\
\text { http://revistas.upel.edu.ve/index.php/si } \\
\text { nopsis_educativa }\end{array}$ & Mejoramiento Profesional del Magisterio \\
\hline 12 & $\begin{array}{l}\text { Investigación y Postgrado } \\
\text { http://revistas.upel.edu.ve/index.php/re } \\
\text { vinpost }\end{array}$ & $\begin{array}{l}\text { Vicerrectorado de Investigación y } \\
\text { Postgrado }\end{array}$ \\
\hline 4 & $\begin{array}{l}\text { investigación y formación } \\
\text { pedagógica } \\
\text { http://revistas.upel.edu.ve/index.php/re } \\
\text { vinvformpedag/index }\end{array}$ & $\begin{array}{l}\text { Extensión San Cristóbal del Instituto de } \\
\text { Mejoramiento Profesional del Magisterio }\end{array}$ \\
\hline 19 & $\begin{array}{l}\text { Laurus } \\
\text { http://revistas.upel.edu.ve/index.php/la } \\
\text { urus }\end{array}$ & Vicerrectorado de Docencia \\
\hline 2 & $\begin{array}{l}\text { Línea Imaginaria } \\
\text { http://revistas.upel.edu.ve/index.php/li } \\
\text { nea_imaginaria }\end{array}$ & $\begin{array}{l}\text { Coordinación General de Investigación } \\
\text { del Instituto Pedagógico Rural Gervasio } \\
\text { Rubio }\end{array}$ \\
\hline 28 & $\begin{array}{l}\text { Paradigma } \\
\text { http://revistas.upel.edu.ve/index.php/p } \\
\text { aradigma }\end{array}$ & $\begin{array}{l}\text { Centro de Investigaciones Educacionales } \\
\text { Paradigma (CIEP) Instituto Pedagógico de } \\
\text { Maracay "Rafael Alberto Escobar Lara }\end{array}$ \\
\hline
\end{tabular}

El trabajo destacó las coincidencias y divergencias para poder interpretar lo que realmente estaba ocurriendo. En este caso, se tomó en cuenta la información proporcionada, pues fueron los interlocutores los que generarían los constructos de las conclusiones de esta investigación.

\section{RESULTADOS}

El conocimiento y saberes relacionados con las TIC construido por la comunidad upelista es un proceso multifactorial y dinámico que implica tanto lo individual como la forma en que el conocimiento se comparte en la colectividad, en donde la 
retroalimentación positiva desde y hacia cada uno de los elementos del sistema es capital.

Se construye el conocimiento a partir de los hallazgos suministrados por los autores en sus productos académicos asumiendo que el investigador ha creado redes, que convergen con las TIC, además que estas están vinculadas a la construcción y reconstrucción del conocimiento que se genera y ha generado en la universidad.

Se valora la información contextualizada en relación con los conocimientos y saberes de las TIC por parte de la comunidad upelista.

A la pregunta si la UPEL considera que son importantes las TIC, se encontró que la respuesta es definitivamente sí. Al respecto Guzmán (2018, 2013, 2008, 2005) reflexiona que se está en la tecnología en todos los ámbitos de la vida $\mathrm{y}$, queramos o no tener acceso a ella, esta sigue avanzando $y$ produciendo cambios a los que la escuela debe responder.

Los tutoriales multimedia, las bases de datos, las bibliotecas electrónicas, los hipertextos distribuidos, entre otros son nuevas maneras de presentar y acceder al conocimiento. En el futuro este tipo de soportes serán utilizados de modo creciente en todos los niveles educativos. Entre las estrategias de enseñanza y aprendizaje aplicadas se encuentran: los foros, los grupos de discusión, los chats, en los que los estudiantes realizan discusiones monitoreadas por el facilitador sobre los diferentes tópicos.

Procesadores de palabras para reportes o informes, hoja de cálculo para tener los datos que muestran las estadísticas del curso o alguna investigación, programas de dibujo, presentaciones con sonido movimiento e imagen, para mostrar nuestros hallazgos entre muchas otras. Usar diferentes aplicaciones para oír la radio, tener acceso a las redes sociales, a nuestro canal favorito de tv, de música o curso en YouTube (Guzmán, 2018 y López V, 2016).

Esto exige competencias que le permiten vivir, tener movilidad, hacer y ser un activo valioso dentro de este entorno cambiante. Lo que crea en él nuevas necesidades de autorrealización, capacitación y formación. El reto del sistema educativo es adaptarse y asumir su función de formarlo permanentemente (Guzmán, 2018 y Castro, Guzmán y Casado, 2007).

El mundo globalizado de hoy exige a la escuela el desarrollo de competencias en TIC en diferentes tipos de organizaciones, especialidades, niveles y modalidades del sistema educativo, en ellos se usan videos, presentaciones entre otros medios $y$ estrategias para la mediación de diferentes contenidos. Quintero (2017); Quintero J. (2017); Castillo (2019); Dilone (2019); Tovar (2019); Alcalá (2019); Hurtado (2019); Rodríguez y Guzmán (2012); Rodríguez y Guzmán (2012); Rodríguez (2008); y Delgado (2019) fueron investigadores que aportaron al presente estudio productos de los postgrados de la UPEL los cuales demostraron que en los perfiles de las diferentes especialidades y en muchas otras profesiones se requiere la incursión y apropiación de las TIC para capacitación pero también para desarrollar sus trabajos, hacer sus estadísticas, cuadros, gráficos, diagramas entre otros y hacer sus presentaciones públicas.

El usuario aprende a conectarse, identificarse, buscar informaciones cada vez más precisas, crear y, posteriormente, definir parámetros de su cuenta, cargar, luego a archivar, contribuir, compartir, pero debe poseer:

1. competencias para manejar las herramientas y las funcionalidades del entorno digital. 
2. la compresión y apropiación de la información y sus contextos

3. Interpretar, decodificar, saber tomar distancia crítica para usar y comprender la información que tomamos de la Red, de las plataformas, de los amigos de las redes sociales, de los tutores pedagógicos $\mathrm{o}$ de varias fuentes institucionales, o no.

Del análisis de los documentos se desprende que las TIC:

1. Facilitan los procesos educativos o no y son base de las competencias que debe desarrollar el ciudadano.

2. Optimizan la interactividad, motivación e intercambio de roles del docente y estudiante adecuando los diseños instruccionales a las necesidades de los usuarios y contenidos con una secuencia y estructura educativa centrada en el aprendizaje y en las características de la audiencia (Suárez y D León, 2007 y 2008).

3. Propician el desarrollo de una gama de hipermedios o multimedia interactivos que proveen de navegación poderosa que permiten al usuario exhibir información enlazada en texto, además representa una interacción multimedio no-textual de porciones de información conectadas, lo cual permite al usuario comenzar desde donde desee, moverse en los alrededores de la aplicación, realizar búsquedas, hojear y tener acceso a módulos interconectados y elementos tales como texto, video, archivos de animaciones, audio y gráficas (Guzmán (2013); De Marco y Guzmán (2005, 2008 y 2012); González y Guzmán (2010);
López y Guzmán (2019); García y Guzmán (2019).

4. Propician el desarrollo de una metodología para la producción de ebook.

5. Sus potencialidades han propiciado la planificación y desarrollo de estrategias innovadoras.

6. Las TIC se integran cuando se emplean para apoyar y ampliar los objetivos curriculares, para motivar y estimular a los estudiantes a entender, comprender mejor y a construir el aprendizaje. No es entonces algo que se hace aisladamente, sino que debe formar parte integral de las diarias actividades que se realizan en el salón de clase.

Sánchez (s/f) plantea que la integración curricular de las TIC implica varias características: a) la utilización y apropiación de las tecnologías, b) el usar las tecnologías para planificar estrategias y facilitar la construcción del aprender, c) el usar las tecnologías para apoyar las actividades en clases, d) integrar las tecnologías como parte del currículum.

\section{CONCLUSIONES}

A modo de conclusión se debe señalar que este estudio develó que en los reglamentos de la UPEL, planes de desarrollo, tesis y trabajos de grado $y$ artículos se contempla la incorporación efectiva de las Tecnologías de Información y Comunicación. Para la universidad estas son importantes como base para dinamizar los procesos de enseñanza y aprendizaje a nivel superior, es por ello que lo incluyen como eje curricular en el diseño 2015. Además, en el introductorio de Extensión se contempla que es para todos y cada uno de los estudiantes que aspiren realizar postgrado en la UPEL. 
Allí se pudo apreciar, entre otros cursos, el de competencias interactivas a través de las Tecnología de Información y Comunicación. Este es un requisito de ingreso presente en el reglamento de Extensión. En los cursos y diplomados de Extensión se exige como conducta de entrada y eje curricular las Tecnologías de Información y Comunicación.

En fin, en la UPEL se han desarrollado hipermedios y multimedios para diferentes asignaturas con conocimientos y saberes variados. Se generaron canales de YouTube y grupos abiertos de Facebook para su uso instruccional y además ha sido temática importante para trabajos de ascenso y artículos.

\section{REFERENCIAS}

Albornoz, J. (1986). El Instituto Pedagógico; una visión retrospectiva. Caracas: Ediciones del Congreso

Alcalá A. (2019). Desarrollo de competencias para la enseñanza del turismo en los docentes de educación comercial de la Unidad Educativa Fe y Alegría Virginia De Ruiz I - La Silsa (trabajo de grado). Instituto Pedagógico de Caracas, Universidad Pedagógica Experimental Libertador, Venezuela

Beltrán, M. (2017). Principios Teóricos Hacia La Integración De Tecnologías De Información $Y$ Comunicación En Formación Docente Para Educación Inicial (tesis doctoral). Instituto Pedagógico de Caracas, Universidad Pedagógica Experimental Libertador, Venezuela

Casado, D., Castro, S. y Guzmán, B. (2007). Las TIC En Los Procesos De Enseñanza Y Aprendizaje. Revista Laurus, 13(23). Recuperado de redalyc.uaemex.mx/pdf/761/76102311.pdf

Castillo E. (2019). Competencias en el Uso Y Manejo De TIC De Los Docentes Del Departamento De Biología Y Química De La UPEL - IPC (trabajo de grado). Instituto Pedagógico de Caracas, Universidad Pedagógica Experimental Libertador, Venezuela
Castro, S. y Guzmán, B. (2001). ¿Es la Tecnología útil en la Educación? Revista de Investigación, 49(1), 11-37

De León C. I. y Suárez J. (2008). El Diseño Instruccional y Tecnologías de la Información y la Comunicación. Posibilidades y Limitaciones. Revista de Investigación, 65, 57-82

De León C, I, y Suárez N, J. (2007). Diseño instruccional y tecnologías de la información y la comunicación: Algunas reflexiones. Revista de Investigación, 31(61), 13-33

De Marco, P. y Guzmán B. (2008). Diseño y evaluación de un prototipo de CD ROM para el Curso Simulaciones y juegos instruccionales del Departamento de Tecnología Educativa en el Instituto Pedagógico de Caracas. Revista de Investigación 65, 149-170. Recuperado de http://www2.scielo.org.ve/pdf/ri/v32n65/ arto8.pdf

De Marco, A. y Guzmán, B. (2005). Evaluación del Curso Simulaciones y Juegos Instruccionales del Departamento de Tecnología Educativa en el Instituto Pedagógico de Caracas (trabajo de ascenso). Instituto Pedagógico de Caracas, Universidad Pedagógica Experimental Libertador, Venezuela

De Marco, A., y Guzmán, B. (Enero - Abril 2012). Un ambiente Web para la asignatura simulaciones y juegos instruccionales de la Universidad Pedagógica Experimental Libertador Revista de Investigación, 75(36). Recuperado de http://www.scielo.org.ve/scielo.php?pid= S101029142012000100006\&script=sci_arttext

Delgado N., (2019). Competencias para el diseño de medios instruccionales apoyados en las TIC, dirigido a los funcionarios de la BPCE "CECILIO ACOSTA (trabajo de grado). Instituto Pedagógico de Caracas, Universidad Pedagógica Experimental Libertador, Venezuela

Dilone, D. (2019). Integración de las Tecnologías de Información y Comunicación en la Educación Inicial (trabajo de grado). Instituto Pedagógico de Caracas, Universidad Pedagógica Experimental Libertador, Venezuela 
García A. y Guzmán B (2018 julio septiembre). Diseño de un curso virtual bajo la plataforma MOODLE. Una visión del autismo desde la Promoción de la Salud Revista Horizontes, 7(2), 181-189. Recuperado de https://www.revistahorizontes.org/index. $\mathrm{php} /$ revistahorizontes/issue/view/11

González, H. y Guzmán B. (2010). Principios Didácticos para la elaboración de un EBook en salud sexual y reproductiva. Revista de Investigación, 71(34), 141-167. Recuperado de http://www2.scielo.org.ve/scielo.php?scri pt=sci_arttext\&pid=S1010291420100003000o8\&lng=es\&nrm=iso

Guzmán, B. (2005). Actitudes de los docentes ante las Tecnologías de Información y Comunicación (tesis doctoral). Instituto Pedagógico de Caracas, Universidad Pedagógica Experimental Libertador, Venezuela

Guzmán, B. (2008). Los docentes de la Universidad Pedagógica Experimental Libertador - Instituto Pedagógico de Caracas ante las Tecnologías de Información y Comunicación. Investigación y postgrado, 1(23), 37-72

Guzmán, B. (2013). Evaluación del Curso Tecnología de Información y Comunicación y Educación Ambiental a Través de sus Productos. (Trabajo de ascenso). Instituto Pedagógico de Caracas, Universidad Pedagógica Experimental Libertador, Venezuela

Guzmán, B. (2016). Departamento de Tecnología Educativa. Una dependencia en cambio permanente. En: Historia de los Departamentos del Instituto Pedagógico de Caracas (375-401). Caracas: Fondo Editorial Mariano Picón Salas del Instituto Pedagógico de Caracas

Guzmán, B. (septiembre 2018). TIC y Medios. [Conferencia] 27 Encuentro Nacional de Profesores de Francés organizado y promovido por la Asociación Venezolana de Profesores de Francés. Pozo de Rosas, Miranda

Hurtado, Y. (2019). Desarrollo instruccional para la innovación educativa, basado en las TIC. Dirigido a los docentes de contabilidad del IPC (trabajo de grado). Instituto Pedagógico de Caracas,
Universidad Pedagógica Experimental Libertador, Venezuela

López, V. E. (2016). Teoría Subyacente en la Promoción y Divulgación de la Educación Ambiental Informal para la Formación del Ciudadano por medio de las Redes Sociales Digitales (tesis doctoral). Instituto Pedagógico de Caracas, Universidad Pedagógica Experimental Libertador, Venezuela

López, E. y Guzmán, B. (2019). Redes sociales y su utilidad en la educación ambiental promoción y divulgación informal. Horizontes. Revista De Investigación En Ciencias de la Educación, 3(12), 249-266. https://doi.org/10.33996/revistahorizonte s.v3i12.85

Niño, A. (2017 Julio - diciembre). Las TIC en la educación universitaria venezolana: una reflexión desde la perspectiva docente Investigación y Formación Pedagógica. Revista del CIEGC, 3(6) 59 67

Ollarves, M. y Hernández, M., y Pirela D. (2018 enero-junio). Quórum Académico, 15(1), 119-131

Oviedo, Y. (2010). Diseños Instruccionales y Tecnologías de Información $y$ Comunicación: una Visión del Docente de la Upel-IPB (tesis doctoral). Instituto Pedagógico de Barquisimeto, Barquisimeto, estado Lara, Universidad Pedagógica Experimental Libertador, Venezuela

Quintero, J. Y. (2017). Competencias Laborales para Optimizar las Funciones de los Supervisores del Área del Call Center de la Empresa PRC333 C.A. (trabajo de grado). Instituto Pedagógico de Caracas, Universidad Pedagógica Experimental Libertador, Venezuela

Quintero, J. Y. (2017). Desarrollo de la Competencia Comunicativa de los Estudiantes de la Asignatura Lengua Española en el Instituto Pedagógico de Caracas (trabajo de grado). Instituto Pedagógico de Caracas, Universidad Pedagógica Experimental Libertador, Venezuela

Rodríguez S., R. (2008). Desarrollo de Competencias Asociadas a los Estudiantes que Ingresan a la Carrera de Profesor en Educación del Instituto Pedagógico de 
Miranda (trabajo de grado). Instituto Pedagógico de Caracas, Universidad Pedagógica Experimental Libertador, Venezuela

Rodríguez S., R. (2016). Modelo Teórico para la Formación del Docente de Música Sustentado en el Desarrollo de Habilidades para el Arreglo Música (tesis doctoral). Instituto Pedagógico de Caracas, Universidad Pedagógica Experimental Libertador, Venezuela

Rodríguez S., Z. (2008). Desarrollo de Competencias Docentes a Través de la Incorporación del Uso de las Tecnologías de la Información y la Comunicación (TIC) en la Especialidad de Educación Musical (tesis doctoral). Instituto Pedagógico de Caracas, Universidad Pedagógica Experimental Libertador, Venezuela

Rodríguez S., Z. (2016). Corpus teórico para la formación del docente de música desde la perspectiva de los valores socioculturales. Caso UPEL (tesis doctoral). Instituto Pedagógico de Caracas, Universidad Pedagógica Experimental Libertador, Venezuela

Rodríguez S., Z. y Guzmán, B. (2012). Competencias en el uso de las TIC en profesores de la especialidad educación musical del Instituto Pedagógico José Manuel Siso Martínez. Revista de Investigación, 436(76)

Ruiz Bolívar, C. (2014). Evaluación de una Experiencia de Tutoría Virtual de Tesis de Grado en el Contexto de un Programa de Doctorado en Educación. PARADIGMA, 35(1), $129-148$

Sánchez, J. (s.f). Departamento de Ciencias de la Computación, Universidad de Chile. Recuperado de http://www.c5.cl/mici/pag/papers/inegr_ curr.pdf

Tovar, Z. (2019). Desarrollo Instruccional en Herramientas Tecnológicas Educativas para los Docentes de Educación Comercial de la Escuela Técnica Robinsoniana y Zamorana Nacional Eduardo Meza Istúriz (trabajo de grado). Instituto Pedagógico de Caracas, Universidad Pedagógica Experimental Libertador, Venezuela
Universidad Pedagógica Experimental Libertador [UPEL] (2003). Recuperado de http://www.upel.edu.ve/infogeneral/Upel/index.htlm

Universidad Pedagógica Experimental Libertador [UPEL] (1985). Políticas de Extensión. Visión Misión Valores. Vicerrectorado de Extensión. Caracas

Universidad Pedagógica Experimental Libertador [UPEL] (1989). Reglamento de Extensión

Universidad Pedagógica Experimental Libertador [UPEL] (1989). Políticas de Investigación. Vicerrectorado de Investigación. Caracas

Universidad Pedagógica Experimental Libertador [UPEL] (1997). La Extensión Universitaria de la UPEL. Vicerrectorado de Extensión. Caracas

Universidad Pedagógica Experimental Libertador [UPEL] (2000). Políticas de Docencia. Vicerrectorado de Docencia. Caracas

Universidad Pedagógica Experimental Libertador [UPEL] (2003). Reglamento de Extensión

Universidad Pedagógica Experimental Libertador [UPEL] (2004). Políticas de Extensión. Visión Misión Valores. Vicerrectorado de Extensión. Caracas

Universidad Pedagógica Experimental Libertador [UPEL] (2007). Plan de Desarrollo 2007 - 2011. Recuperado de http://150.187.142.20/sede/Planificacion/P lanDesarrollo20072010/Plan\%2ode\%2oDesarrollo.pdf

Universidad Pedagógica Experimental Libertador [UPEL] (2007). Plan de Desarrollo 2007 - 2013. Recuperado de http://150.187.142.20/sede/Planificacion/P lanDesarrollo20072013/Plan\%2ode\%2oDesarrollo.pdf

Universidad Pedagógica Experimental Libertador [UPEL] (2007). Plan de Desarrollo 2014 - 2018. Recuperado de http://150.187.142.20/sede/Planificacion/P lanDesarrollo20142018/Plan\%2ode\%2oDesarrollo.pdf

Villarroel, A, Carpio, E. y Castillo M. (2014 mayo - agosto). Revista de Investigación, $38(82)$ 